\title{
Mecanismos de patogénesis del melanoma maligno
}

\author{
Mechanisms of pathogenesis of malignant melanoma
}

\section{Catalina Restrepo', Margarita María Velásquez ${ }^{2}$}

1. Médica, residente de Dermatología, Universidad de Antioquia, Medellín, Colombia

2. Médica dermatóloga; profesora, Sección de Dermatología, Centro de Investigaciones Dermatológicas, CIDERM, Universidad de Antioquia, Medellín, Colombia

\section{Resumen}

El melanoma es una de las neoplasias malignas de la piel más agresivas y peligrosas. Tiene múltiples factores de riesgo, siendo uno de los más importantes la exposición crónica e intermitente al sol. Además, tiene un componente genético que se asocia a los casos familiares, debido principalmente a las mutaciones del gen $C D K N 2 A$; sin embargo, la mayoría de los melanomas son esporádicos. En su patogenia se describen mutaciones en la línea germinal y mutaciones somáticas, las cuales modifican la regulación del ciclo celular y permiten un crecimiento no regulado. El sistema inmunitario juega un papel muy importante en la patogénesis porque, a pesar de que este tumor tiene antígenos que son claramente reconocidos, permitiendo que el sistema inmunitario monte una respuesta, las células del melanoma logran evadir la inmunidad innata y la adaptativa, para así invadir y generar metástasis.

Palabras Clave: melanoma, inmunología, fisiología, genética.

\section{Summary}

Melanoma is one of the most aggressive and dangerous malignant neoplasms of the skin. It has multiple risk factors; one of the most important is the chronic and intermittent exposure to sunlight. It also has a genetic component associated with familial cases that is mainly due to CDKN2A mutations, but the majority of melanomas are sporadic. In its pathogenesis mutations have been described in the germ line and also somatic mutations, which alter the cell cycle regulation and allow unregulated growth. The immune system plays an important role in the pathogenesis because although this tumor has antigens that are clearly recognized, allowing the system to trigger an immune response, melanoma cells manage to evade the innate and adaptive immunity in order to invade and metastasize.

KEY WORDS: Melanoma, immunology, physiology, genetics.

\section{Correspondencia:}

Catalina Restrepo

Email: catar.r@hotmail.com

Recibido: 27 de agosto de 2011.

Aceptado: 25 abril de 2012.

No se reportan conflictos de intereses.

\section{Introducción}

El melanoma maligno es una de las neoplasias cutáneas más peligrosas y agresivas. Según diferentes reportes, se encuentra entre la quinta y la décima causas de neoplasia en América y es la forma más común de neoplasia maligna fatal en los adultos jóvenes ${ }^{1}$. Se observó un incremento de 3,1 \% por año en su incidencia en los Estados Unidos, desde 1992 a 2004². En Europa, el incre- mento oscila entre 3 y $7 \%$ por año. En Australia y Nueva Zelanda es donde se presenta el mayor número de casos $\mathrm{y}$, en general, se duplica la incidencia cada 10 años ${ }^{3}$. El riesgo de que a una persona a lo largo de su vida se le diagnostique melanoma se estimaba previamente en 1 de 6oo, en 1965, y 1 de 150, en 19854,5. En el reporte del 2008, se calculó que para el 2010, 1 de cada 50 personas en los Estados Unidos desarrollaría melanoma 6 . 
La incidencia se ha triplicado en las últimas dos décadas en la población de raza blanca. Se estima que en el 2009 se diagnosticaron 68.720 nuevos casos de melanoma (38.080 en hombres y 29.640 en mujeres) ${ }^{7}$. Aunque el melanoma sólo representa el $4 \%$ de todos los cánceres de piel, es responsable, aproximadamente, de 70 a $80 \%$ de las muertes por cáncer de piel.

El incremento de la incidencia de melanoma ha tenido múltiples explicaciones. Algunos se han atribuido a una mejor aproximación diagnóstica a los pacientes con lesiones de piel, un mayor número de biopsias y la mayor habilidad para la interpretación histológica; otros lo han atribuido a una mayor tamización y, finalmente, otro grupo lo explica por la mayor exposición a los factores de riesgo.

En Colombia, un reporte de 1978 a 1982 reveló que la incidencia anual de melanoma en Colombia había sido de 3 por 100.000 en mujeres y de 3,3 por 100.000 en hombres. En el Instituto Nacional de Cancerología, en el año 2000, se registraron 22 muertes por melanoma cutáneo que correspondían al $2 \%$ de todas las muertes por cáncer en ese año en la institución ${ }^{8}$. En un estudio epidemiológico de cáncer de piel en el Centro Dermatológico Federico Lleras Acosta de Bogotá, entre 2003 y 2005, se encontró que los casos de melanoma pasaron de 2,7 por 10.000 en el 2003, a 13 por 10.000 en el $2005^{9}$. En el registro de población de cáncer de Cali, de 2003 a 2007 , se reportó en hombres un número de casos de 168 , con una tasa de 3,5 por 100.000 personas por año $\mathrm{y}$, en mujeres, 172 casos con una tasa de 3,1 por 100.000 personas por $a \tilde{n} 0^{10}$. En este mismo registro también se encuentra un reporte de la mortalidad por cáncer de piel de 2004 a 2008, la cual presentó una tasa 2,2 por 100.000 personas por año en hombres y una tasa de 1,2 por 100.000 por año en mujeres, pero no se diferencia entre melanoma y cáncer no melanoma ${ }^{11}$. En GLOBOCAN del 2008, reportaron una incidencia del melanoma en Colombia de 1,9 por 100.000 habitantes, con una mortalidad de 0,5 por $100.000^{12}$.

\section{Factores de riesgo}

Se describen dos grupos grandes de factores de riesgo para desarrollar melanoma, ambientales y genéticos, lo que explica la diferencia entre las diferentes razas, ubicaciones geográficas y culturas ${ }^{13}$.

La exposición a la radiación ultravioleta (UV) se considera el factor ambiental más relacionado con el desarrollo del melanoma maligno, y los rayos solares UVB (de 290 a $320 \mathrm{~nm}$ ) son los más importantes.

Aunque no se ha demostrado experimentalmente una relación causal directa entre la exposición a la radiación
UV y el melanoma, varios estudios indirectos han demostrado que es un factor de riesgo mayor; entre estos, la revisión sistemática de la literatura científica publicada en $1997^{14}$, demostró que la exposición intermitente e intensa a la radiación solar desde la niñez es un factor de riesgo independiente que, además, se encuentra influenciada por la posición geográfica, con mayor impacto en aquellas regiones sobre la línea ecuatorial, y por el tipo de piel.

El melanoma maligno se ha asociado con otras enfermedades, entre ellas, la endometriosis. Un estudio epidemiológico prospectivo mostró un incremento significativo de melanoma en mujeres con endometriosis (riego relativo de 1,6) ${ }^{15}$. Asimismo, en un estudio que de 14.00o pacientes con enfermedad de Parkinson, se encontró un incremento significativo en la incidencia de melanoma, comparada con la de la población general $\left(\mathrm{OR}=1,95 ; \mathrm{IC}_{95 \%} 2,4-2,6\right)^{16}$.

La cantidad de nevos típicos se relaciona con la exposición solar ${ }^{17}$ y con el riesgo de desarrollar melanoma, según diferentes autores, más de 250 más de 50 nevos ${ }^{18,19}$.

Los nevos congénitos, así como la historia personal, juegan un papel importante y es claro que una persona con antecedentes de melanoma tiene mayor riesgo de desarrollar un segundo melanoma cutáneo primario ${ }^{20}$.

Aproximadamente, el $10 \%$ de todos los melanomas son de presentación familiar. En algunos estudios se ha encontrado implicada la mutación del gen $C D K N 2 A$ en la generación del melanoma ${ }^{21}$. Se han relacionado defectos moleculares en los genes supresores tumorales y oncogenes, con los casos de melanoma de tipo familiar. El gen supresor tumoral con mayor implicación hace parte del cromosoma 9p y es conocido como CDKN2A, p16INK4A o MTS1 ${ }^{21}$.

Los nevos atípicos, anteriormente llamados displasia melanocítica, se han considerado lesiones precancerosas. Los nevos atípicos se han asociado con un incremento entre 3 y 20 veces del riesgo de desarrollar melanoma maligno, en comparación con la población general ${ }^{22}$. Estos nevos y el melanoma se han asociado con mutaciones en la línea germinal de $C D K N 2 A, A R F, C D K 4$ y mutaciones somáticas en los genes PTEN y $B R A F^{23}$.

Finalmente, uno de los factores implicados en el riesgo de melanoma es la inmunosupresión de cualquier causa, bien sea la infección por virus de la inmunodeficiencia humana o el tratamiento con inmunosupresores, como en los pacientes con trasplantes, en quienes se ha observado que la recurrencia de melanoma es tan alta como $19 \%$ en algunas series ${ }^{24}$. En la Tabla 1 se resumen los factores de riesgo asociados al melanoma

Dada la elevada morbimortalidad del melanoma ma- 


\begin{tabular}{cc}
\hline Factores & Riesgo Relativo \\
\hline Fuerte historia familiar (3 o más familiares con melanoma) & $35-70$ \\
Débil historia familiar & 3 \\
Nevos benignos múltiples & 11 \\
Nevos atípicos múltiples & 11 \\
Melanoma previo & 8.5 \\
Otro cáncer de piel previo & 2.9 \\
Receptores de trasplantes & 3 \\
SIDA & 1.5 \\
Piel fototipo I (se quema, no broncea) & 1.7 \\
Pecas & 2.5 \\
Endometriosis & 1.6 \\
Pelo rojo & 2.4 \\
\hline Quemaduras solares de segundo grado & 2.5 \\
\hline
\end{tabular}

TABLA 1. Factores de riesgo para melanoma maligno

ligno y el incremento de su incidencia, es importante ampliar nuestros conocimientos en los factores de riesgo y los cambios inmunológicos, lo cual permitiría una mejor comprensión de la enfermedad y contribuir al desarrollo de estrategias efectivas para la prevención y el tratamiento.

\section{Patogénesis}

La inmunología y la biología del melanoma pueden explicar por qué algunos tumores permanecen estables o silentes durante años, por qué otros presentan agresividad desmesurada, y, también, por qué algunos tumores que tenían un pronóstico desfavorable con base en los índices de Clark y Breslow, no presentan problemas mientras que otros con muy buen pronóstico inicial desarrollan un comportamiento muy agresivo, con invasión y metástasis.

Al expresar múltiples moléculas, el melanoma va adquiriendo la capacidad de crecimiento local y a distancia, induciendo alteraciones vasculares y en la matriz extracelular y, a la vez, alterando la respuesta inmunitaria que trata de contener su expansión. Resulta esencial la vigilancia que ejerce el sistema inmunitario sobre las células tumorales del melanoma para controlar las neoplasias incipientes. La capacidad metastásica podría ser consecuencia de los mecanismos que esas células tumorales desarrollan para escapar del control inmunológico (GráfICA 1).

De todos los tumores, el melanoma es el que tiene el mayor índice de regresión espontánea total, y se aprecia regresión parcial en $13 \%$ de los casos ${ }^{25}$. Actualmente, se acepta que la respuesta celular normal mediada por los linfocitos $\mathrm{T}$ es la principal implicada en la destrucción de los tumores ya establecidos. Sin embargo, es posible que en las etapas iniciales de la diseminación, los anticuerpos anticélulas tumorales puedan ejercer un papel regulador, esencial para evitar esa diseminación.

A continuación se describen algunos aspectos genéticos e inmunológicos que contribuyen a la evasión de la respuesta inmunitaria por parte de las células del melanoma.

\section{Genética}

El melanoma es el resultado de la acumulación de múltiples mutaciones en el genoma del melanocito, que llevan a que sufra alteración del ciclo celular y proliferación desmedida, evasión de la respuesta inmunitaria, invasión del tumor y metástasis.

Las mutaciones en los genes de vulnerabilidad ante el cáncer varían en su grado de penetrancia. El gen de alta penetrancia más caracterizado es $C D K N 2 A$ (inhibidor de las cinasas dependiente de ciclina A2); éste se encuentra en el cromosoma 9p21 y codifica dos proteínas diferentes, las cuales predisponen al melanoma, p16INK4a y p14ARF. De 25 a $40 \%$ de los casos de melanoma de tipo familiar portan mutación en el gen $C D K N 2 A$ inactivo, que depende de la cinasa ciclina $4\left(\mathrm{CDK}_{4}\right)$ y está ubicado en el cromosoma 12q14. $C D K_{4}$ es otro gen de vulnerabilidad de alta penetrancia en el melanoma ${ }^{26}$. El gen $C D K N 2 A$, 
INMUNOPATOGÉNESIS DEL MELANOMA

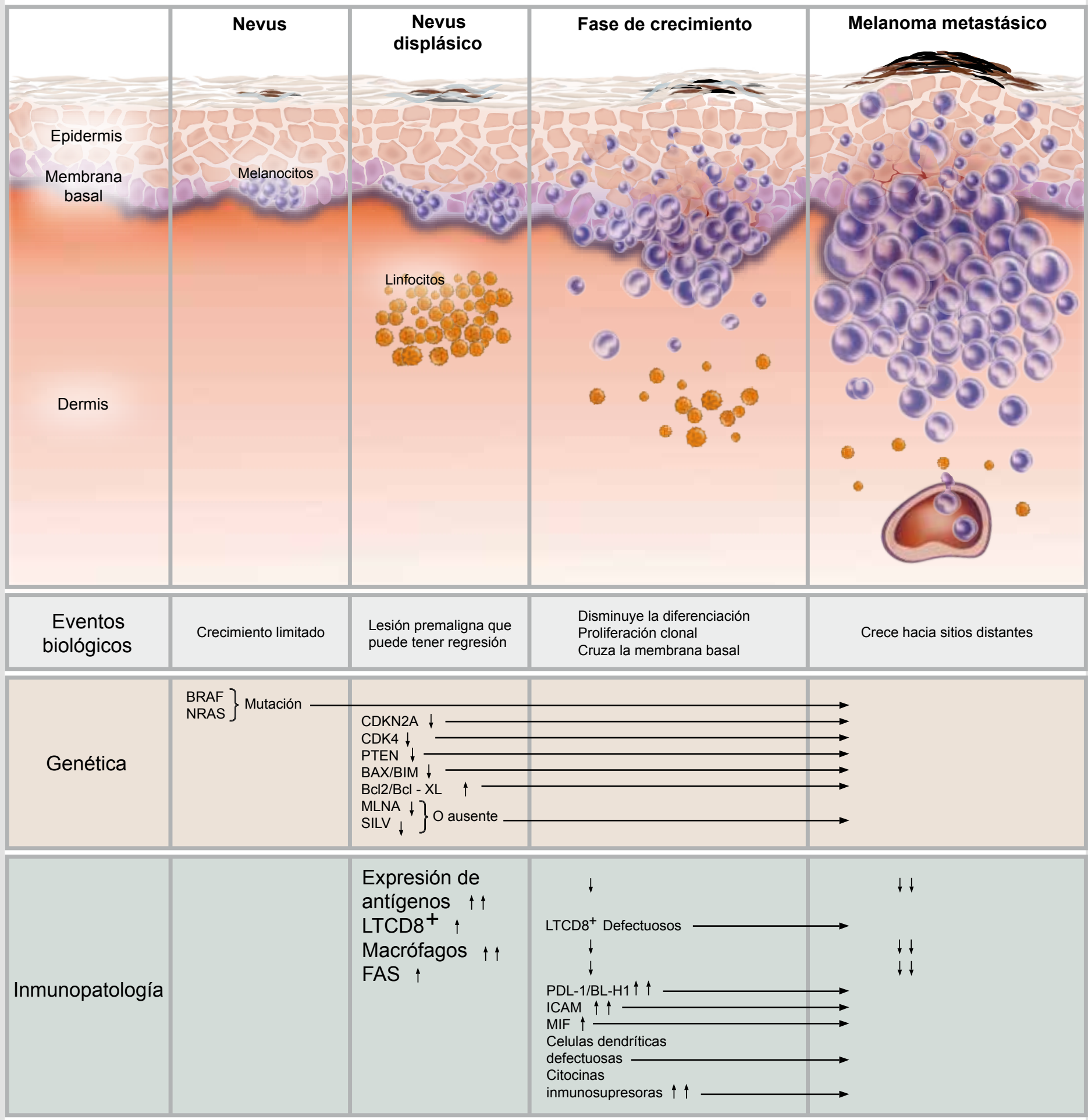

Mutaciones en BRAF y NRAS, implicadas en el desarrollo y progresión del melanoma. CDKN2A, CDK4 y PTEN, la pérdida de estos produce activación de la proliferación celular sin control. Bax/Bim, moléculas pro-apotóticas, que están disminuidas en el melanoma desde etapas iniciales. Bcl2/Bcl-XL moléculas antiapoptóticas aumentadas desde estadios tempranos. SILV y MLANA, genes cuya expresión esta disminuida o ausente en la progresión de nevos a melanoma. Antígenos, su expresión en el melanoma esta aumentada en etapas iniciales, pero con la progresión esta expresión disminuye permitiendo evadir la respuesta inmune. LTCD8+,en melanomas ya establecidos son incapaces de liberar perforinas y otras enzimas citotóxicas. Macrófagos, están en mayor cantidad en melanomas no metastásicos. FAS y PDL1/B7-H1, su expresión va disminuyendo según la progresión del melanoma para poder escapar de la apoptosis. ICAM-1, molécula de adhesión que tiene mayor expresión en células tumorales que en nevos, que se correlaciona directamente con el estadio tumoral. MIF, mayormente expresada en células tumorales, inhibiendo la migración de macrófagos y estimulando el crecimiento y la invasión. Células dendríticas, son defectuosas al no expresar adecuadamente moléculas coestimuladoras, produciendo una regulación a la baja de LT. Citocinas inmunosupresoras, están aumentadas para promover un ambiente inmunosupresor. 
mediante la interacción con el $C D K_{4}$, actúa como un inhibidor específico de la CDK4/ciclina D cinasa y como un potencial regulador negativo de la proliferación celular. La proteína p16INK4a se une a CDK4/6 e inhibe su interacción con la ciclina $\mathrm{D}$ que, de otro modo, estimula el paso a través del G1 a S mediante la inactivación del gen supresor de tumor retinoblastoma ${ }^{26,27}$.

El gen $C D K 4$ codifica la ciclina dependiente de la cinasa, la activación de las mutaciones en $C D K_{4}$, permite el paso sin control G1/S, que conduce a la proliferación celular incontrolada y al crecimiento del tumor ${ }^{26,27}$. melanomas esporádicos, estos son más frecuentes $(85$ a 90\%). Entre las vías de señalización alteradas en los melanomas esporádicos, se describen las siguientes ${ }^{26}$ :

La vía MAPK (Ras/Raf/MEK/ERK, cinasas de proteínas activadas por mitógenos) se encuentra en numerosos tipos de células y es un importante regulador del crecimiento y de la supervivencia celular. Las mutaciones que resultan en la activación de la vía MAPK se encuentran en $90 \%$ de los melanomas y, por lo general, ocurren en los oncogenes $B R A F$ y $N R A S$, que permanecen activados estimulando constantemente la proliferación y la senescencia celular ${ }^{28}$. Las alteraciones en la señalización mediante la vía MAPK están implicadas en el desarrollo y la progresión del melanoma.

El oncogén NRAS puede activar la vía MAPK o la vía $\mathrm{PI}_{3} \mathrm{~K}$, necesarias para las señales de supervivencia celular. Las mutaciones pueden ser más comunes en los sitios de exposición crónica al sol y se producen al principio de la tumorogénesis ${ }^{29}$; se encuentran en 10 a $20 \%$ de los melanomas. Las mutaciones de NRAS también son comunes en los tumores benignos de novo ${ }^{30}$. El NRAS parece estimular la proliferación de melanocitos.

En el oncogén $B R A F$ se ha identificado una mutación somática, que está presente en $66 \%$ de los melanomas malignos $^{31}$. No obstante, esta mutación también se ha encontrado en muchos lesiones benignas ${ }^{32}$, lo que sugiere que la activación de $B R A F$ puede ser necesaria para la proliferación melanocítica, pero no es suficiente por sí misma para la formación de tumores; además, se ha demostrado que una deficiencia concomitante de p53 también puede ser necesaria para obtener tumorogénesis $^{28,33}$. El BRAF juega un papel importante en las vías de señalización para la activación de los factores de transcripción; activa MEK, que influye en la actividad de BCL, y ERK, que estimula la proliferación y MITF.

El gen MITF influye en el desarrollo y la supervivencia de los melanocitos y actúa como un factor de transcripción clave para la regulación de la expresión de las proteínas mayores melanogénicas, incluyendo TYR y las proteínas relacionadas con TYR. Se ha demostrado que promueven la supervivencia de los melanocitos al aumentar la expresión de la proteína antiapoptótica
BCL-2. Por otra parte, los bajos niveles de expresión de MITF se correlacionan con un mal pronóstico en el melanoma, por su papel en la diferenciación de las células del melanoma. Los melanomas en los cuales las células están más diferenciadas, con mayor expresión de MITF exhiben menor agresividad. Del 10 al $20 \%$ de los melanomas presentan amplificación de MITF, con una mayor incidencia en las lesiones metastásicas ${ }^{26,34}$. El MITF también regula la transcripción de genes SLIV (melanocyte-specific genes silver homologue) y MLANA (melan-A). La disminución o ausencia de la expresión de SILV y MLANA acompañan la progresión de nevos a melanoma y, además, empeoran el pronóstico ${ }^{34}$.

El gen supresor tumoral PTEN tiene múltiples actividades que incluyen la regulación del sistema embrionario, la adhesión, la migración y la apoptosis; además, está implicado en la diferenciación y el crecimiento de células madre ${ }^{35}$. El PTEN codifica fosfatasas que desfosforilan PIP3 a PIP2, disminuyendo la actividad de la vía $\mathrm{PI}_{3} \mathrm{~K}$ que llevaría finalmente a proliferación celular. Con la pérdida de PTEN se activa la vía de $\mathrm{PI}_{3} \mathrm{~K}$, lo cual se encuentra en $60 \%$ de los melanomas ${ }^{36}$. Se han identificado mutaciones somáticas de PTEN en melanomas y en metástasis ${ }^{29,37}$. En varios estudios se ha demostrado que la restauración de PTEN reduce las metástasis y la generación de tumores ${ }^{38}$

El c-kit es un protooncogén que codifica el receptor de membrana factor de tirosina cinasa, al cual se unen factores de crecimiento, que se encuentran en numerosos tipos de células, incluyendo los melanocitos. Las señales del $c$-kit utilizan la vía MAPK y su objetivo es la transcripción de $M I T F^{39}$. Recientemente, se encontraron mutaciones oncogénicas, aumento de las copias de c-kit, o ambas cosas, en $39 \%$ de los melanomas de las mucosas, en $36 \%$ de los de zonas distales, y en el $28 \%$ de los de la piel dañada por exposición crónica al sol, en 102 tumores primarios ${ }^{40}$. El c-kit está implicado de forma preferencial en el melanoma lentiginoso "acral” y los melanomas de las mucosas; estas variantes son menos frecuentes en el mundo, pero en Latinoamérica la variedad lentiginosa "acral” es más frecuente que otros tipos de melanoma.

En el melanoma, se observa un desequilibrio entre genes de la familia BCL-2/Bax. Los melanocitos expresan constitutivamente BCL-2, el cual es el principal inhibidor de apoptosis. Se ha observado un aumento de la expresión de la proteína BCL-2 en el melanoma maligno, en comparación con los melanocitos y los nevos, al contrario que el Bax y el Bim, que son proteínas proapoptóticas, cuya expresión está disminuida en los melanomas con respecto a los nevos ${ }^{26,41}$.

También se ha visto un incremento en la expresión de $B c l-X L$ (otro gen antiapoptótico) en diversas mues- 


\begin{tabular}{cll}
\hline GEN O PROTEINA & \multicolumn{1}{c}{ FUNCIÓN } & \multicolumn{1}{c}{ CAMBIOS EN EL MELANOMA } \\
\hline CDKN2A & Regulador de la proliferación celular & $\begin{array}{l}\text { Mutaciones de la línea germinal en algunos melanomas } \\
\text { familiares }\end{array}$ \\
CDK4 & Promotor de la proliferación celular & $\begin{array}{l}\text { Mutaciones en la línea germinal, permiten el paso sin } \\
\text { control G1 / S, que conduce a la proliferación celular } \\
\text { descontrolada y al crecimiento del tumor }\end{array}$ \\
N-RAS- BRAF & Oncogén & $\begin{array}{l}\text { Mutaciones somáticas. Estimula constantemente la } \\
\text { proliferación y la senescencia celular. }\end{array}$ \\
MITF & $\begin{array}{l}\text { Influye en el desarrollo y la superviven- } \\
\text { cia de los melanocitos y actúa como un } \\
\text { factor de transcripción }\end{array}$ & $\begin{array}{l}\text { Mutación somática. } \\
\text { Aumenta la expresión de Bcl-2, inhibiendo la apoptosis }\end{array}$ \\
PTEN & Supresor de tumor, suprime a Pl3K & $\begin{array}{l}\text { Delección esporádica. Activándose Pl3K, que lleva a la } \\
\text { proliferación celular. }\end{array}$ \\
C-KIT & $\begin{array}{l}\text { Proto-oncogén } \\
\text { El objetivo es la transcripción MITF }\end{array}$ & $\begin{array}{l}\text { Mutaciones oncogénicas y / o aumentos de las copias } \\
\text { de KIT }\end{array}$ \\
Bcl-2 / Bcl-XL & Antiapoptóticos & Se observado un aumento de la expresión \\
Bax / Bim & Pro-apoptóticos & Su expresión esta disminuida \\
\hline \hline
\end{tabular}

TABLA 2. Mutaciones asociadas a melanoma.

tras de melanoma que, incluso, se ha demostrado en los melanomas en sus estadios iniciales y, quizá, esta expresión prematura esté asociada con la transformación maligna y la capacidad invasiva y metastásica ${ }^{42}$. En la Tabla 2 se presentan los aspectos genéticos asociados al melanoma.

\section{Inmunología}

La capacidad de las células tumorales para evadir la respuesta del sistema inmunológico se conoce como "escape tumoral”. Constantemente se están formando células tumorales que en su mayoría son eliminadas por el sistema inmunitario. Sin embargo, a medida que se van seleccionando variedades tumorales que no pueden ser eliminadas, se puede formar un tumor sólido capaz de desencadena señales de inflamación que dan lugar al reclutamiento de células del sistema inmunitario innato, entre ellas, las células dendríticas; posteriormente, se reclutan linfocitos que infiltran y pueden eliminar el tumor o permanecer en equilibrio con las células tumorales, de tal manera que éstas no mueren ni proliferan. El tumor puede permanecer en estado de equilibrio por varios años. Las células tumorales pueden adquirir la capacidad de escapar del ataque inmunitario y crecer, invadir y producir metástasis ${ }^{43}$.

El melanoma tiene múltiples mecanismos para escapar exitosamente de la inmunovigilancia; ya se han mencionado algunas alteraciones genéticas que permiten que crezca e invada. A continuación, se describe cómo el melanoma puede evadir los diferentes componentes del sistema inmunitario.

Los antígenos que se han encontrado asociados al melanoma son muy importantes, puesto que permiten que el sistema inmunitario reconozca las células tumorales y pueda montar una respuesta. El melanoma cutáneo primario es capaz de desencadenar una respuesta inmunitaria, como se infiere por la intensa reacción linfoide que suele acompañar dichas lesiones. Por el contrario, dicha capacidad se pierde en gran medida en las metástasis. Los principales antígenos expresados en la superficie celular del melanoma están asociados predominantemente al HLA de clase I, y están implicados en la regulación de la proliferación celular, diferenciación, control de la pigmentación y calidad de la melanina ${ }^{44,45}$. Los antígenos de diferenciación melanocítica son proteínas normales que se expresan en melanocitos normales y melanomas, en el $90 \%{ }^{46}$. Las proteínas que actúan como antígenos en el melanoma son la gp10o, una proteína expresada en la membrana del melanosoma frecuentemente reconocida por los linfocitos T citotóxicos ${ }^{47}$, y Melan A/MART-1, una proteína transmembrana sin sitios de $\mathrm{N}$-glucosilación, altamente enriquecida en los melanosomas tempranos. Se expresa en la piel, la retina, y en la mayoría de los melanocitos y melanomas en cultivo ${ }^{48}$.

Los antígenos oncofetales, son otro grupo de antí- 
genos cuya expresión generalmente está restringida a células tumorales o células de la línea germinal. Entre los antígenos oncofetales están las proteínas llamadas MAGE, codificadas por una familia de 24 genes, que se clasifican en tres subfamilias y se encuentran en regiones diferentes del cromosoma $X^{49,50}$. La familia MAGE juega un rol fisiológico y patológico durante la embriogénesis mediante un mecanismo de activación y desactivación de genes por metilación. En el caso de la formación de un tumor, estos genes podrían ser reactivados y, las proteínas resultantes, reconocidas y atacadas por el sistema inmunitario. De aquí se desprende que las proteínas MAGE desempeñan un rol importante en la vigilancia inmunitaria de ciertos tipos de tumores ${ }^{50}$.

El NY-ESO-1, otro antígeno oncofetal, está expresado en 20 a $40 \%$ de los melanomas y en cerca de $50 \%$ de los pacientes con tumores avanzados que expresan NYESO-1; es posible detectar anticuerpos y linfocitos $\mathrm{T}$ CD8+ espontáneos específicos contra este antígeno. Se ha demostrado que es uno de los más inmunógenos ${ }^{49,51}$.

Esta diversidad de antígenos asociados al melanoma se ha aprovechado para la elaboración de distintas estrategias de vacunación en pacientes con enfermedad declarada, pero no para prevenir la enfermedad, buscando lograr una inmunización eficaz del paciente al inducir una respuesta humoral y celular específica en suficiente magnitud como para neutralizar el crecimiento y la diseminación de las células de melanoma que ya pudieran haber hecho metástasis ${ }^{52}$. También, se están utilizando linfocitos reactivos contra los antígenos asociados a melanoma como tratamiento adoptivo. En estos casos, los linfocitos antígeno-específicos son inyectados en grandes volúmenes, ya sea a partir de linfocitos $\mathrm{T}$ infiltrantes de tejido o generados a partir de linfocitos de sangre periférica transducidos con receptores de células, en combinación con IL-2 ${ }^{53}$.

Uno de los principales mecanismos para evadir la respuesta inmunitaria en el melanoma es la falta de activación de los linfocitos $\mathrm{T}$, dada por la pérdida de HLA I en la superficie de las células tumorales, con pérdida total o de baja expresión de uno o de varios alelos HLA I, variantes alélicas mutadas o fallas en la señalización celular ${ }^{54}$. Esto le permite a las células tumorales escapar de la destrucción por parte de las linfocitos T citotóxicos. Además de este hecho, la propia diversidad antigénica dentro del tumor puede impedir la generación de una respuesta inmunitaria antitumoral eficaz, ya que las células que no expresan los antígenos tumorales escapan del reconocimiento por los linfocitos T citotóxicos específicos $^{54}$. También, se ha descrito la disminución de HLA II en las células presentadoras de antígenos y la falta de señales coestimuladoras B7; esto lleva a entorpecer la función adecuada de los linfocitos $\mathrm{T} \mathrm{CD}_{4}+$, lo que re- sulta en anergia de los linfocitos específicos y tolerancia inmunológica al tumor ${ }^{55}$.

Otros factores expresados en las células del melanoma pueden inhibir las células T, tales como PD-L1/B7-H1, que actúan sobre las células $\mathrm{T}$ regulando su activación y diferenciación, al unirse a PD-1 expresados en los linfocitos T. PD-1/B7-H1 promueve la producción de IL-10 e induce apoptosis de los linfocitos T, media la inhibición de la respuesta de los linfocitos $\mathrm{T}$, probablemente inhibiendo la progresión del ciclo celular por medio de motivos ITIM intracitoplásmicos que tienen los PD-1 de los linfocitos $\mathrm{T}$, desencadenando señales inhibidoras dentro de la célula ${ }^{56}$.

La pérdida de moléculas específicas de adhesión, como, por ejemplo, ICAM-1, en algunos melanomas y precisamente en los casos de melanoma maligno, conlleva a mal pronóstico. La ICAM-1 es un tipo de molécula intercelular de adherencia presente continuamente en concentraciones bajas en las membranas de leucocitos y de células endoteliales. La ICAM-1 es un ligando para LFA-1 (integrina), pero también se ha visto que la ICAM-1 es expresada significativamente en mayor proporción en melanomas que en nevos. Existe relación entre el nivel de expresión de ICAM-1 y el grosor tumoral; además, los niveles séricos solubles de ICAM-1 se encuentran elevados en pacientes con melanoma, lo cual se relaciona directamente con el estadio tumoral e inversamente con la supervivencia en los estadios II y III ${ }^{57}$.

Los linfocitos CD8+ (células citotóxicas) podrían jugar un rol protector en el melanoma; no obstante, se ha observado que en los pacientes con melanoma ya establecido, los linfocitos T DC8+ son incapaces de liberar perforinas y otras enzimas líticas necesarias para la actividad citotóxica ${ }^{58}$.

El papel de las células B en la inmunidad tumoral aún no está claro. Según los hallazgos, se admite que pueden tener un papel en el reclutamiento de los mastocitos de estroma del tumor. Se ha propuesto que las células B juegan un papel en la linfangiogénesis y las metástasis de las células del melanoma ${ }^{59}$.

En el melanoma también se ve alterada la función de los macrófagos. El rango del contenido de macrófagos es de o a $30 \%$. En las lesiones metastásicas, todos los melanomas contienen menos de $10 \%$ de macrófagos, mientras que, en los no metastáticos, se encuentra en mayor cantidad de macrófagos ${ }^{6}$. Por lo tanto, parece que hay una relación inversa entre el contenido de los macrófagos de tumores y su capacidad de hacer metástasis.

Los macrófagos activados se subdividen en dos grupos principales, M1 y M2. Los macrófagos M1 son capaces de producir iNOS (oxido nítrico sintasa inducible), IL-12 y el factor de necrosis tumoral alfa (TNF- $\alpha$ ). Los macrófagos M2 producen arginasa, IL-10, el factor 
de crecimiento transformante beta (TGF beta) y la prostaglandina E2 (PGE2), con su respectivo repertorio de citocinas. Los M1 promueven la eliminación del tumor, mientras que los macrófagos M2 promueven la angiogénesis y la remodelación de tejidos.

Los macrófagos M2 contribuyen a un ambiente permisivo para el tumor, mediante la restricción de la respuesta de tipo 1 y la secreción de IL-10. Esta acción perpetúa un estado $\mathrm{M}_{2}{ }^{61}$.

Las líneas celulares de melanoma pueden secretar proteína quimiotáctica de monocitos (MCP-1), pero no la normal que secretan los melanocitos. Los bajos niveles de MCP-1 pueden dar lugar a la formación de tumores por la poca filtración de macrófagos. Por otra parte, los altos niveles de MCP-1 se asocian con secreción masiva de monocitos e infiltración de macrófagos en la masa tumoral, lo que lleva a su destrucción en unos pocos días y puede ayudar al crecimiento tumoral estimulado por los monocitos y macrófagos, probablemente debido al aumento de la angiogénesis ${ }^{60}$.

Además, se ha visto que las células tumorales tienen una expresión mayor que los melanocitos normales de factor inhibidor de la migración de macrófagos (MIF), la cual es una citocina multifuncional. El MIF puede inhibir la migración de los macrófagos, modula la respuesta inmunitaria, inhibe factores de crecimiento y factores angiogénicos y la actividad de las células NK. Por lo tanto, mantiene un microambiente de privilegio inmunitario de las células tumorales ${ }^{62}$. Como tal, el MIF puede estimular el crecimiento y la invasión de forma concomitante, del melanoma con la formación de nuevos vasos sanguíneos. Esta molécula tiene acción carcinogénica en el melanoma por el hecho de que es un potente modulador de las vías de p53 y E2F-dependiente. Este último se activa en respuesta a la señalización oncogénica, lo que permite que el ciclo celular no tenga ningún control ${ }^{63}$.

Otra forma que tiene el melanoma para escapar de los macrófagos es la llamada actividad inhibitoria del melanoma (MIA), mediada por la secreción de una molécula. Esta es una pequeña proteína que interactúa con las proteínas de la matriz extracelular. Es producida por las células del melanoma y puede inhibir su adhesión a la proteína extracelular de la matriz (fibronectina) ${ }^{64}$. La transformación maligna de los melanocitos a melanoma se relaciona con la regulación al alza de la expresión de la MIA. La producción de MIA facilita la progresión del tumor y el desarrollo de las potencialidades metastásicas en el melanoma, inhibiendo la adhesión de las células tumorales a la matriz extracelular (fibronectina) y, por lo tanto, mejorar su potencial invasión.

Factores solubles producidos por los macrófagos estimulan a las células del melanoma para aumentar la producción de MIA lo que promueve la capacidad de invasión y la migración celular ${ }^{64,65}$.

Un papel importante de las células dendríticas es la expresión de moléculas coestimuladoras y la producción de citocinas durante la diferenciación de las células $\mathrm{T}$ ayudadoras después de la presentación de antígenos. Estos eventos se caracterizan por las moléculas coestimuladoras CD40, CD80 y CD86. Las células dendríticas en el melanoma no expresan adecuadamente las moléculas coestimuladoras, produciendo un perfil de tolerancia y una regulación a la baja de los linfocitos T. Se ha demostrado que las células del melanoma son capaces de inhibir la diferenciación de los primeros precursores de células de Langerhans in vitro. El grado de inhibición se relacionó directamente con la agresividad de la línea de melanoma ${ }^{60,66}$. De hecho, en algunos melanomas invasivos se ha encontrado completa ausencia de las células dendríticas ${ }^{66}$. Se ha encontrado que la densidad de células dendríticas maduras, especialmente cuando están en presencia de células $\mathrm{T}$ activadas, podría ser un factor predictor de la supervivencia y un parámetro de la respuesta inmunitaria funcional en pacientes con melanoma ${ }^{67}$.

Se sabe que el melanoma genera un perfil de citocinas con propiedades inmunológicas inhibidoras, las cuales promueven un ambiente localmente inmunosupresor que le permitirá el crecimiento y la progresión. Entre las citocinas inmunosupresoras secretadas por el melanoma, están TGF-B, IL-6, IL-8, IL-10 y la enzima inmunosupresora IDO (indolamina 2,3-dioxigenasa) ${ }^{67,68}$. La IL-10 es una citocina que inhibe la acción de los macrófagos y de las células dendríticas, la respuesta de la inmunidad del huésped, y la síntesis de IL-12 por macrófagos, la cual es un estímulo para la secreción de IFN gamma; además, inhibe la expresión de coestimuladores y de moléculas HLA de clase II. La expresión de IL-6 indica un mal pronóstico en el melanoma, pues aumenta la angiogénesis por medio de VEGF y Cox2, y estimula el crecimiento ${ }^{60}$. El TGF-B inhibe la proliferación y activación de linfocitos ${ }^{69}$. La IDO es una enzima responsable del catabolismo del triptófano, aminoácido esencial para la proliferación de las células T; al promover la anergia y la muerte celular y contribuira la generación de células $\mathrm{T}$ reguladoras tiene un rol en la tolerancia inmunológica al tumor ${ }^{68}$.

El melanoma no sólo evita el reconocimiento por el sistema inmunitario, sino que, además, altera activamente las funciones del sistema inmunitario en el microambiente local. Dicha inmunosupresión incluye la presencia de linfocitos $\mathrm{T}$ reguladores ${ }^{70}$ y la modulación de las células dendríticas del microambiente tumoral, como ya se ha mencionado, influyendo en su maduración y otorgándoles un perfil de tolerancia. Se ha repor- 


\begin{tabular}{|c|c|c|c|}
\hline $\begin{array}{l}\text { MOLÉCULAS } \\
\text { CÉLULAS }\end{array}$ & FUNCIÓN & HALLAZGOS EN EL MELANOMA & RESULTADO \\
\hline HLA I & $\begin{array}{l}\text {-Presentación de antígenos a } \\
\text { los linfocitos T }\end{array}$ & $\begin{array}{l}\text { Pérdida de HLA I en la superficie de } \\
\text { las células tumorales }\end{array}$ & Presentación antigénica deficiente \\
\hline B7 & -Coestimulación & Ausencia de B7 & $\begin{array}{l}\text { Anergia de los linfocitos y tolerancia } \\
\text { inmunológica }\end{array}$ \\
\hline $\mathrm{PD}-\mathrm{L}_{1} / \mathrm{B}_{7}-\mathrm{H}_{1}$ & $\begin{array}{l}\text {-Actúan sobre las células T } \\
\text { regulando su activación y } \\
\text { diferenciación }\end{array}$ & $\begin{array}{l}\text { Expresados en las células del } \\
\text { melanoma }\end{array}$ & $\begin{array}{l}\text { Apoptosis de los linfocitos T, media } \\
\text { la inhibición de la respuesta de los } \\
\text { linfocitos T }\end{array}$ \\
\hline ICAM-1 & $\begin{array}{l}\text {-Molécula intercelular de } \\
\text { adherencia }\end{array}$ & $\begin{array}{l}\text { Puede estar expresado en mayor } \\
\text { o en menor proporción en el } \\
\text { melanoma }\end{array}$ & $\begin{array}{l}\text { Se correlaciona directamente con el } \\
\text { estadio tumoral e inversamente con la } \\
\text { supervivencia en los estadios II y III }\end{array}$ \\
\hline Linfocitos CD8+ & $\begin{array}{l}\text {-Células citotóxicas } \\
\text {-inmunidad adaptativa }\end{array}$ & $\begin{array}{l}\text { Incapaces de liberar perforinas y } \\
\text { otras enzimas líticas }\end{array}$ & Escape tumoral a la respuesta inmune \\
\hline Células B & $\begin{array}{l}\text {-Células productoras de } \\
\text { anticuerpos } \\
\text {-Inmunidad adaptativa }\end{array}$ & Aún no es claro & $\begin{array}{l}\text { Linfangiogenesis y metástasis de las } \\
\text { células del melanoma }\end{array}$ \\
\hline Macrófagos & $\begin{array}{l}\text {-Fagocitar } \\
\text {-Inmunidad innata }\end{array}$ & $\begin{array}{l}\text { Todos los melanomas contienen } \\
\text { menos del } 10 \% \text { de macrófagos }\end{array}$ & Metástasis \\
\hline MCP-1 & $\begin{array}{l}\text {-Proteína quimiotáctica de } \\
\text { monocitos }\end{array}$ & Secretada en poca cantidad & $\begin{array}{l}\text { Formación tumoral por la poca filtración } \\
\text { de macrófagos }\end{array}$ \\
\hline MIF & $\begin{array}{l}\text {-Inhibe la migración de } \\
\text { macrófagos. } \\
\text {-Modula la respuesta inmune. } \\
\text { Inhibe las células NK. }\end{array}$ & Expresión mayor & $\begin{array}{l}\text { Mantiene un microambiente de privilegio } \\
\text { inmune de las células tumorales }\end{array}$ \\
\hline MIA & $\begin{array}{l}\text {-Proteína que interactúa con las } \\
\text { proteínas de matriz extracelular }\end{array}$ & $\begin{array}{l}\text { Producida por las células del } \\
\text { melanoma }\end{array}$ & $\begin{array}{l}\text { Facilita la progresión del tumor y el } \\
\text { desarrollo de metástasis }\end{array}$ \\
\hline Células dendríticas & -Presentadoras de antígenos & $\begin{array}{l}\text { No expresan adecuadamente las } \\
\text { moléculas coestimuladoras }\end{array}$ & $\begin{array}{l}\text { Perfil tolerogénico, y una regulación a la } \\
\text { baja de los linfocitos T }\end{array}$ \\
\hline $\begin{array}{l}\text { Perfil de citocinas } \\
\text { TGF B, IL-6, IL-8, } \\
\text { IL-10 e IDO }\end{array}$ & -Citocinas inmunoinhibitorias & $\begin{array}{l}\text { Producidas por las células } \\
\text { melanociticas }\end{array}$ & $\begin{array}{l}\text { Promueve un ambiente localmente } \\
\text { inmunosupresor, para crecimiento y } \\
\text { progresión }\end{array}$ \\
\hline Células NK & $\begin{array}{l}\text {-Papel contra células } \\
\text { tumorales, por medio de } \\
\text { perforinas y producción de IFN } \\
\text { gamma }\end{array}$ & $\begin{array}{l}\text { Las células del melanoma tienen } \\
\text { una expresión aumentada de HLA-G }\end{array}$ & Inhibición la actividad de las células NK. \\
\hline
\end{tabular}

TABla 3. Mecanismos de escape a la respuesta inmune en melanoma.

tado que el microambiente inmunosupresor también es generado por el tumor primario en los ganglios centinelas con metástasis o sin ellas, lo cual sugiere la hipótesis de que células dendríticas con perfil de tolerancia en el tumor primario, migran a los ganglios centinelas, donde secretan IDO y TGF-B, creando un microambiente privilegiado que condiciona previamente a los ganglios para la subsecuente diseminación del melanoma ${ }^{71}$.

Las NK son células muy importantes que actúan en la inmunidad innata y que tienen un papel fundamental en la respuesta contra células tumorales, por medio de de perforinas y producción de IFN gamma ${ }^{72}$.

Las células del melanoma pueden tener una regulación a la baja de las moléculas HLA de clase I, lo que aumenta la actividad de las células NK, pero esto por sí solo no es suficiente para erradicar las células tumorales ${ }^{73}$.

Las células NK participan en la vigilancia del tumor mediante una variedad de mecanismos y receptores. El $\mathrm{NKG}_{2} \mathrm{D}$ es un receptor que aparece en las células NK y en un subconjunto de células $\mathrm{T}$ (delta-gamma y $\mathrm{T}$ alfa- 
beta). Es un receptor de reconocimiento primario de las células sospechosas, tales como las células tumorales. Sin embargo, las células tumorales que expresan NKG2D pueden escapar de la vigilancia, quizá mediante supresión de la síntesis de perforina o resistencia a la misma ${ }^{74}$. Se ha demostrado que las células del melanoma pueden tener una expresión aumentada de HLA-G, una molécula HLA no clásica, y de esta forma, inhibir la actividad de las células NK mediante la interacción con KIR ${ }^{75}$.

Otro mecanismo que induce la eliminación de células $\mathrm{T}$ específicas es la inducción de apoptosis, debido a la expresión de FasL ${ }^{76}$. Por otro lado, las células tumorales pueden perder la expresión de Fas y volverse resistente a la apoptosis. En la TABLA 3 se resumen los factores inmunológicos asociados a melanoma.

\section{Conclusión}

Se presentaron los aspectos genéticos e inmunológicos más importantes, descritos a la fecha, que participan en la patogénesis del melanoma. El estudio y la comprensión de la inmunopatogénesis podrían permitir el futuro desarrollo de herramientas de detección y tratamientos más eficaces que aumenten la supervivencia de estos pacientes.

\section{Referencias}

1. Balch CM, Gershenwald JE, Soong S-J, Thompson JF, Atkins MB, Byrd DR, et al. Final version of 2009 AJCC melanoma staging and classification. J. Clin. Oncol. 2009;27:6199-206.

2. Linos E, Swetter SM, Cockburn MG, Colditz GA, Clarke CA. Increasing burden of melanoma in the United States. J. Invest. Dermatol. 2009;129:1666-74.

3. Whiteman DC, Valery P, McWhirter W, Green AC. Risk factors for childhood melanoma in Queensland, Australia. Int. J. Cancer. 1997;70:26-31.

4. Jemal A, Murray T, Samuels A, Ghafoor A, Ward E, Thun MJ. Cancer statistics, 2003. CA Cancer J Clin. 2003;53:5-26.

5. Edwards BK, Brown ML, Wingo PA, Howe HL, Ward E, Ries LAG, et al. Annual report to the nation on the status of cancer, 1975 2002, featuring population-based trends in cancer treatment. J. Natl. Cancer Inst. 2005;97:1407-27.

6. Jemal A, Siegel R, Ward E, Hao Y, Xu J, Murray T, et al. Cancer statistics, 2008. CA Cancer J Clin. 2008;58:71-96.

7. Jemal A, Siegel R, Ward E, Hao Y, Xu J, Thun MJ. Cancer statistics, 2009. CA Cancer J Clin. 2009;59:225-49.

8. Acosta ÁE, Fierro E, Velásquez VE, Rueda X. Melanoma: patogénesis, clínica e histopatología. (Spanish). Revista de la Asociación Colombiana de Dermatología y Cirugía Dermatológica. 2009;17:87-108.

9. Nova-Villanueva J, Sánchez-Vanegas G, De Quintana LP. Cáncer de Piel: Perfil Epidemiológico de un Centro de Referencia en Colombia 2003-2005. (Spanish). Revista de Salud Pública. 2007 dic;9:595-601.
10. Universidad del Valle. Registro Poblacional de Cáncer de Cali, Colombia: 2003-2007. [Internet]. 2008 [citado 2011 nov 27]. Available a partir de: http://rpcc.univalle.edu.co/es/incidencias/ Estadisticas/index.php

11. Universidad del Valle. Registro Poblacional de Cáncer de Cali, Colombia : 2004-2008 [Internet]. 2010 [citado 2011 nov 27]. Available a partir de: http://rpcc.univalle.edu.co/es/incidencias/ Estadisticas/index.php

12. GLOBOCAN. Cancer incidence and mortality worldwide in 2008 [Internet]. 2010 [citado 2010 oct 14]. Available a partir de: http:// globocan.iarc.fr/factsheet.asp

13. Rhodes AR, Weinstock MA, Fitzpatrick TB, Mihm MC Jr, Sober AJ. Risk factors for cutaneous melanoma. A practical method of recognizing predisposed individuals. JAMA. 1987;258:3146-54.

14. Elwood JM, Jopson J. Melanoma and sun exposure: an overview of published studies. Int. J. Cancer. 1997;73:198-203.

15. Kvaskoff M, Mesrine S, Fournier A, Boutron-Ruault M-C, ClavelChapelon F. Personal history of endometriosis and risk of cutaneous melanoma in a large prospective cohort of French women. Arch. Intern. Med. 2007;167:2061-5.

16. Olsen JH, Friis S, Frederiksen K, McLaughlin JK, Mellemkjaer L, Møller H. Atypical cancer pattern in patients with Parkinson's disease. Br. J. Cancer. 2005;92:201-5.

17. Harrison SL, MacLennan R, Speare R, Wronski I. Sun exposure and melanocytic naevi in young Australian children. Lancet. 1994;344:1529-32.

18. Bataille V, Bishop JA, Sasieni P, Swerdlow AJ, Pinney E, Griffiths $\mathrm{K}$, et al. Risk of cutaneous melanoma in relation to the numbers, types and sites of naevi: a case-control study. Br. J. Cancer. 1996;73:1605-11.

19. Olsen CM, Carroll HJ, Whiteman DC. Estimating the attributable fraction for cancer: A meta-analysis of nevi and melanoma. Cancer Prev Res (Phila). 2010;3:233-45.

20. Bradford PT, Freedman DM, Goldstein AM, Tucker MA. Increased risk of second primary cancers after a diagnosis of melanoma. Arch Dermatol. 2010;146:265-72.

21. Chaudru V, Chompret A, Bressac-de Paillerets B, Spatz A, Avril M-F, Demenais F. Influence of genes, nevi, and sun sensitivity on melanoma risk in a family sample unselected by family history and in melanoma-prone families. J. Natl. Cancer Inst. 2004;96:785-95.

22. Clinically atypical moles predict melanoma: A prospective st...: Melanoma Research [Internet]. [citado 2012 may 29]. Available a partir de: http://journals.lww.com/melanomaresearch/ Fulltext/1993/0300o/Clinically_atypical_moles_predict_ melanoma_A.216.aspx

23. Celebi JT, Ward KM, Wanner M, Polsky D, Kopf AW. Evaluation of germline CDKN2A, ARF, CDK4, PTEN, and BRAF alterations in atypical mole syndrome. Clin. Exp. Dermatol. 2005;30:68-70.

24. Penn I. Malignant melanoma in organ allograft recipients. Transplantation. 1996;61:274-8.

25. Kelly JW, Sagebiel RW, Blois MS. Regression in malignant melanoma. A histologic feature without independent prognostic significance. Cancer. 1985;56:2287-91.

26. Ibrahim N, Haluska FG. Molecular pathogenesis of cutaneous melanocytic neoplasms. Annu Rev Pathol. 2009;4:551-79. 
27. Chudnovsky Y, Khavari PA, Adams AE. Melanoma genetics and the development of rational therapeutics. J. Clin. Invest. 2005;115:813-24.

28. Hocker TL, Singh MK, Tsao H. Melanoma genetics and therapeutic approaches in the 21st century: moving from the benchside to the bedside. J. Invest. Dermatol. 2008;128:2575-95.

29. Singh M, Lin J, Hocker TL, Tsao H. Genetics of melanoma tumorigenesis. Br. J. Dermatol. 2008;158:15-21.

30. Bauer J, Curtin JA, Pinkel D, Bastian BC. Congenital melanocytic nevi frequently harbor NRAS mutations but no BRAF mutations. J. Invest. Dermatol. 2007;127:179-82.

31. Davies H, Bignell GR, Cox C, Stephens P, Edkins S, Clegg S, et al. Mutations of the BRAF gene in human cancer. Nature. 2002;417:949-54.

32. Pollock PM, Harper UL, Hansen KS, Yudt LM, Stark M, Robbins $\mathrm{CM}$, et al. High frequency of BRAF mutations in nevi. Nat. Genet. 2003;33:19-20.

33. Patton EE, Widlund HR, Kutok JL, Kopani KR, Amatruda JF, Murphey RD, et al. BRAF mutations are sufficient to promote nevi formation and cooperate with $\mathrm{p}_{53}$ in the genesis of melanoma. Curr. Biol. 2005;15:249-54.

34. Thompson JF, Scolyer RA, Kefford RF. Cutaneous melanoma in the era of molecular profiling. Lancet. 2009;374:362-5.

35. Yamada KM, Araki M. Tumor suppressor PTEN: modulator of cell signaling, growth, migration and apoptosis. J. Cell. Sci. 2001;114:2375-82.

36. Stahl JM, Sharma A, Cheung M, Zimmerman M, Cheng JQ, Bosenberg MW, et al. Deregulated Akt3 activity promotes development of malignant melanoma. Cancer Res. 2004;64:7002-10.

37. Tsao H, Goel V, Wu H, Yang G, Haluska FG. Genetic interaction between NRAS and BRAF mutations and PTEN/MMAC1 inactivation in melanoma. J. Invest. Dermatol. 2004;122:337-41.

38. Wu H, Goel V, Haluska FG. PTEN signaling pathways in melanoma. Oncogene. 2003;22:3113-22.

39. Wu M, Hemesath TJ, Takemoto CM, Horstmann MA, Wells AG, Price ER, et al. c-Kit triggers dual phosphorylations, which couple activation and degradation of the essential melanocyte factor Mi. Genes Dev. 2000;14:301-12.

40. Curtin JA, Busam K, Pinkel D, Bastian BC. Somatic activation of KIT in distinct subtypes of melanoma. J. Clin. Oncol. 2006;24:4340-6.

41. Dai DL, Wang Y, Liu M, Martinka M, Li G. Bim expression is reduced in human cutaneous melanomas. J. Invest. Dermatol. 2008;128:403-7.

42. Tang L, Tron VA, Reed JC, Mah KJ, Krajewska M, Li G, et al. Expression of apoptosis regulators in cutaneous malignant melanoma. Clin. Cancer Res. 1998;4:1865-71.

43. Dunn GP, Bruce AT, Ikeda H, Old LJ, Schreiber RD. Cancer immunoediting: from immunosurveillance to tumor escape. Nat. Immunol. 2002;3:991-8.

44. Castelli C, Rivoltini L, Andreola G, Carrabba M, Renkvist N, Parmiani G. T-cell recognition of melanoma-associated antigens. J. Cell. Physiol. 2000;182:323-31.

45. Griffioen M, Kessler JH, Borghi M, van Soest RA, van der Minne $\mathrm{CE}$, Nouta J, et al. Detection and functional analysis of CD8+ T cells specific for PRAME: a target for T-cell therapy. Clin. Cancer Res. 2006;12:3130-6.
46. Bennett DC. Human melanocyte senescence and melanoma susceptibility genes. Oncogene. 2003;22:3063-9.

47. Kawakami Y, Eliyahu S, Delgado CH, Robbins PF, Rivoltini L, Topalian SL, et al. Cloning of the gene coding for a shared human melanoma antigen recognized by autologous $\mathrm{T}$ cells infiltrating into tumor. Proc. Natl. Acad. Sci. U.S.A. 1994;91:3515-9.

48. Coulie PG, Brichard V, Van Pel A, Wölfel T, Schneider J, Traversari $\mathrm{C}$, et al. A new gene coding for a differentiation antigen recognized by autologous cytolytic $\mathrm{T}$ lymphocytes on HLA-A2 melanomas. J. Exp. Med. 1994;180:35-42.

49. Boon T, Coulie PG, Van den Eynde BJ, van der Bruggen P. Human $\mathrm{T}$ cell responses against melanoma. Annu. Rev. Immunol. 2006;24:175-208

50. Xiao J, Chen H-S. [Biological functions of melanoma-associated antigens (MAGEs) in cell activities]. Ai Zheng. 2005;24:124-8.

51. Stockert E, Jäger E, Chen YT, Scanlan MJ, Gout I, Karbach J, et al. A survey of the humoral immune response of cancer patients to a panel of human tumor antigens. J. Exp. Med. 1998;187:1349-54.

52. Eggermont AMM, Schadendorf D. Melanoma and immunotherapy. Hematol. Oncol. Clin. North Am. 2009;23:547-64.

53. Krönig H, Hofer K, Conrad H, Guilaume P, Müller J, Schiemann $\mathrm{M}$, et al. Allorestricted T lymphocytes with a high avidity T-cell receptor towards NY-ESO-1 have potent anti-tumor activity. Int. J. Cancer. 2009;125:649-55.

54. Carretero R, Romero JM, Ruiz-Cabello F, Maleno I, Rodriguez F, Camacho FM, et al. Analysis of HLA class I expression in progressing and regressing metastatic melanoma lesions after immunotherapy. Immunogenetics. 2008;60:439-47.

55. Hersey P, Si Z, Smith MJ, Thomas WD. Expression of the costimulatory molecule $\mathrm{B} 7$ on melanoma cells. Int. J. Cancer. 1994;58:527-32.

56. Dong H, Strome SE, Salomao DR, Tamura H, Hirano F, Flies DB, et al. Tumor-associated $\mathrm{B} 7-\mathrm{H} 1$ promotes T-cell apoptosis: a potential mechanism of immune evasion. Nat. Med. 2002;8:793-800.

57. Natali PG, Hamby CV, Felding-Habermann B, Liang B, Nicotra MR Di Filippo F, et al. Clinical significance of alpha(v)betaz integrin and intercellular adhesion molecule-1 expression in cutaneous malignant melanoma lesions. Cancer Res. 1997;57:1554-6o.

58. Mortarini R, Piris A, Maurichi A, Molla A, Bersani I, Bono A, et al. Lack of terminally differentiated tumor-specific CD8+ T cells at tumor site in spite of antitumor immunity to self-antigens in human metastatic melanoma. Cancer Res. 2003;63:2535-45.

59. Harrell MI, Iritani BM, Ruddell A. Tumor-induced sentinel lymph node lymphangiogenesis and increased lymph flow precede melanoma metastasis. Am. J. Pathol. 2007;170:774-86.

6o. Hussein MR. Tumour-associated macrophages and melanoma tumourigenesis: integrating the complexity. Int J Exp Pathol. 2006;87:163-76.

61. Moore KW, de Waal Malefyt R, Coffman RL, O'Garra A. Interleukin-10 and the interleukin-10 receptor. Annu. Rev. Immunol. 2001;19:683-765.

62. Repp AC, Mayhew ES, Apte S, Niederkorn JY. Human uveal melanoma cells produce macrophage migration-inhibitory factor to prevent lysis by NK cells. J. Immunol. 2000;165:710-5.

63. Petrenko O, Moll UM. Macrophage migration inhibitory factor MIF interferes with the Rb-E2F pathway. Mol. Cell. 2005;17:225-36. 
64. Bosserhoff AK, Buettner R. Expression, function and clinical relevance of MIA (melanoma inhibitory activity). Histol. Histopathol. 2002;17:289-300.

65. Callejo SA, Marshall J-C, Cools-Lartigue J, Saraiva VS, Burnier MN Jr. Macrophage-derived soluble factor enhances melanoma inhibitory activity expression by uveal melanoma cells in vitro. Melanoma Res. 2004;14:91-5.

66. Stene MA, Babajanians M, Bhuta S, Cochran AJ. Quantitative alterations in cutaneous Langerhans cells during the evolution of malignant melanoma of the skin. J. Invest. Dermatol. 1988;91:125-8.

67. Ladányi A, Kiss J, Somlai B, Gilde K, Fejos Z, Mohos A, et al. Density of DC-LAMP(+) mature dendritic cells in combination with activated $\mathrm{T}$ lymphocytes infiltrating primary cutaneous melanoma is a strong independent prognostic factor. Cancer Immunol. Immunother. 2007;56:1459-69.

68. Chen PW, Mellon JK, Mayhew E, Wang S, He YG, Hogan N, et al. Uveal melanoma expression of indoleamine 2,3-deoxygenase: establishment of an immune privileged environment by tryptophan depletion. Exp. Eye Res. 2007;85:617-25.

69. Derynck R, Akhurst RJ, Balmain A. TGF-beta signaling in tumor suppression and cancer progression. Nat. Genet. 2001;29:117-29.
70. Jandus C, Bioley G, Speiser DE, Romero P. Selective accumulation of differentiated $\mathrm{FOXP}_{3}(+) \mathrm{CD}_{4}(+) \mathrm{T}$ cells in metastatic tumor lesions from melanoma patients compared to peripheral blood. Cancer Immunol. Immunother. 2008;57:1795-805.

71. Polak ME, Borthwick NJ, Gabriel FG, Johnson P, Higgins B, Hurren $\mathrm{J}$, et al. Mechanisms of local immunosuppression in cutaneous melanoma. Br. J. Cancer. 2007;96:1879-87.

72. Zamai L, Ponti C, Mirandola P, Gobbi G, Papa S, Galeotti L, et al. NK cells and cancer. J. Immunol. 2007;178:4011-6.

73. Pardoll DM. Immunology. Stress, NK receptors, and immune surveillance. Science. 2001;294:534-6.

74. Hayakawa Y, Kelly JM, Westwood JA, Darcy PK, Diefenbach A, Raulet $\mathrm{D}$, et al. Cutting edge: tumor rejection mediated by NKG2D receptor-ligand interaction is dependent upon perforin. J. Immunol. 2002;169:5377-81.

75. Paul P, Rouas-Freiss N, Khalil-Daher I, Moreau P, Riteau B, Le Gal FA, et al. HLA-G expression in melanoma: a way for tumor cells to escape from immunosurveillance. Proc. Natl. Acad. Sci. U.S.A. 1998;95:4510-5.

76. Hahne M, Rimoldi D, Schröter M, Romero P, Schreier M, French LE, et al. Melanoma cell expression of Fas(Apo-1/CD95) ligand: implications for tumor immune escape. Science. 1996;274:1363-6. 